\title{
Physical activity and risk of cognitive decline: a meta-analysis of prospective studies
}

\author{
- F. Sofi ${ }^{1,2,3}$, D. Valecchi ${ }^{1}$, D. Bacci ${ }^{1}$, R. Abbate ${ }^{2}$, G. F. Gensini ${ }^{1}$, A. Casini ${ }^{3}$ \& C. Macchi ${ }^{1}$ \\ From the ${ }^{1}$ Don Carlo Gnocchi Foundation, Centro S. Maria agli Ulivi, Onlus IRCCS; ${ }^{2}$ Department of Medical and Surgical Critical Care, Thrombosis \\ Centre, University of Florence; and ${ }^{3}$ Regional Agency of Nutrition, Azienda Ospedaliero-Universitaria Careggi, Florence, Italy
}

\begin{abstract}
Sofi F, Valecchi D, Bacci D, Abbate R, Gensini GF, Casini A, Macchi C (Centro S. Maria agli Ulivi, Onlus IRCCS; Thrombosis Centre, University of Florence; Azienda Ospedaliero-Universitaria Careggi, Florence, Italy) Physical activity and risk of cognitive decline: a meta-analysis of prospective studies. J Intern Med 2010; doi: 10.1111/j.1365-2796.2010. 02281.x.
\end{abstract}

Objective. The relationship between physical activity and cognitive function is intriguing but controversial. We performed a systematic meta-analysis of all the available prospective studies that investigated the association between physical activity and risk of cognitive decline in nondemented subjects.

Methods. We conducted an electronic literature search through MedLine, Embase, Google Scholar, Web of Science, The Cochrane Library and bibliographies of retrieved articles up to January 2010. Studies were included if they analysed prospectively the association between physical activity and cognitive decline in nondemented subjects.

Results. After the review process, 15 prospective studies (12 cohorts) were included in the final analysis.
These studies included 33816 nondemented subjects followed for 1-12 years. A total of 3210 patients showed cognitive decline during the follow-up. The cumulative analysis for all the studies under a random-effects model showed that subjects who performed a high level of physical activity were significantly protected (-38\%) against cognitive decline during the follow-up (hazard ratio (HR) 0.62, 95\% confidence interval (CI) 0.54-0.70; $P<0.00001$ ). Furthermore, even analysis of low-to-moderate level exercise also showed a significant protection (-35\%) against cognitive impairment (HR 0.65, 95\% CI 0.57$0.75 ; P<0.00001)$.

Conclusion. This is the first meta-analysis to evaluate the role of physical activity on cognitive decline amongst nondemented subjects. The present results suggest a significant and consistent protection for all levels of physical activity against the occurrence of cognitive decline.

Keywords: cognitive decline, dementia, exercise, physical activity.

\section{Introduction}

It is unquestionable that physical activity has positive effects on health; indeed, over the last few decades, a large body of evidence has shown that physical activity helps to reduce the risk of cardiovascular and cerebrovascular diseases, diabetes, obesity, hypertension and some cancers [1]. Moreover, it has been demonstrated that an active lifestyle impacts on all causes of mortality. With ageing, some cognitive functions such as attention, memory and concentration decline, becoming slower and inefficient, as for some physical functions such as walking and balance. These manifestations are the result of neural cell loss in the frontal, parietal and temporal lobes [2] and strongly depend on an ipofunction of the monoaminergic and cholinergic pathways [3]. Many of these cognitive changes are evident and can cause mild disability, even if a state of dementia is not reached.

Cognitve decline is heterogeneous, depending on various factors. Many studies have shown an inverse relation between physical activity and the risk of developing cognitive decline [4, 5], but the cause of the association has not been clearly established. Individuals who remain active throughout life, especially during middle age, generally have better cognitive 
performance during later life, so preserving their cognitive functions for longer. Recent evidence suggests that in addition to reducing vascular risk factors, physical activity may increase directly the production of neurotrophic factors in the brain [6].

The results of a recent meta-analysis showed that physical exercise is able to reduce the incidence of neurodegenerative diseases; in particular, dementia and Alzheimer's disease [7]. By contrast, few and conflicting data are available on the possible protective role of physical activity on the occurrence of cognitive decline, independent of the onset of neurodegenerative disease [8-18].

Therefore, the aim of this study was to conduct a meta-analysis of all the available prospective cohort studies that investigated the association between physical activity and cognitive decline in nondemented subjects.

\section{Methods \\ Selection of studies}

Studies that investigated the possible association between physical activity and cognitive decline in nondemented adults were identified through a computerized search of all electronic databases: MedLine (source: PubMed, 1966 to January 2010), Embase (1980 to January 2010), Web of Science, The Cochrane Library (source: The Cochrane Central Register of Controlled Trials, 2009, issue 1), Clinicaltrials.org and Google Scholar. Relevant keywords relating to physical activity as Medical Subject Heading terms and text words ('physical activity' or 'physical exercise' or 'exercise', or 'fitness' or 'training') were used in combination with words relating to cognitive impairment ('cognitive decline' or 'cognitive function', or 'cognitive impairment' or 'cognitive loss', or 'dementia', or 'cognition' or 'memory'). We limited the search strategy to prospective cohort epidemiological studies, with nolanguage restrictions, supplemented by manually reviewing the reference list of all retrieved articles.

Two investigators (FS, DV) assessed all potentially relevant articles for eligibility. The decision to include or exclude studies was hierarchical and made on the basis of the following: (i) the study title; (ii) the study abstract; and (iii) the complete study manuscript. In the event of conflicting opinions between investigators, disagreement was resolved through discussion.
Eligible studies were included if they met all of the following criteria: (i) a prospective cohort design; (ii) the association between physical activity and cognitive function as the primary or secondary outcome; (iii) nondemented subjects evaluated at baseline; (iv) clear definitions of methods used to assess cognitive performance and cognitive decline; (v) reported data on physical activity levels in relation to cognitive function; and (vi) reported estimates of association between physical activity and cognitive decline. Accordingly, studies were excluded if: (i) the design was cross-sectional, case control or interventional; (ii) outcomes other than those of interest for the metaanalysis were considered; (iii) patients with dementia or cognitive decline at baseline were included in the study; (iv) the association between physical activity and cognitive decline was not reported; or (v) estimates of the association between physical activity and the decline in cognitive function were not presented (Data S1).

The outcome of interest for the current meta-analysis was cognitive decline or cognitive impairment, defined as decline in cognitive functioning tests at follow-up examination (see Table 1 for further information about tests used to measure cognitive function).

\section{Data extraction}

All data were reviewed and separately extracted by two independent investigators (FS and DV) using a standardized form. The following patient characteristics were recorded: data and study cohort, country of the study cohort, baseline year, number of subjects at baseline, gender of the cohort, years of follow-up, age of the study cohort at baseline, definition of outcome of interest, methods used to assess cognitive function and physical activity, hazard ratio (HR) and confidence interval (CI) values for risk of cognitive decline, and adjustment for confounding factors in multivariate models.

\section{Statistical analysis}

We used Review Manager (RevMan; version 5.0.23 for Macintosh; Copenhagen, Denmark) to pool results from the individual studies.

Pooled results are reported as $\mathrm{HR}$ and are presented with 95\% CI with two-sided $P$ values using a randomeffects model (DerSimonian and Laird method). $P<0.05$ was considered to be statistically significant. When available, we used the results of the original studies from multivariate models with the most 


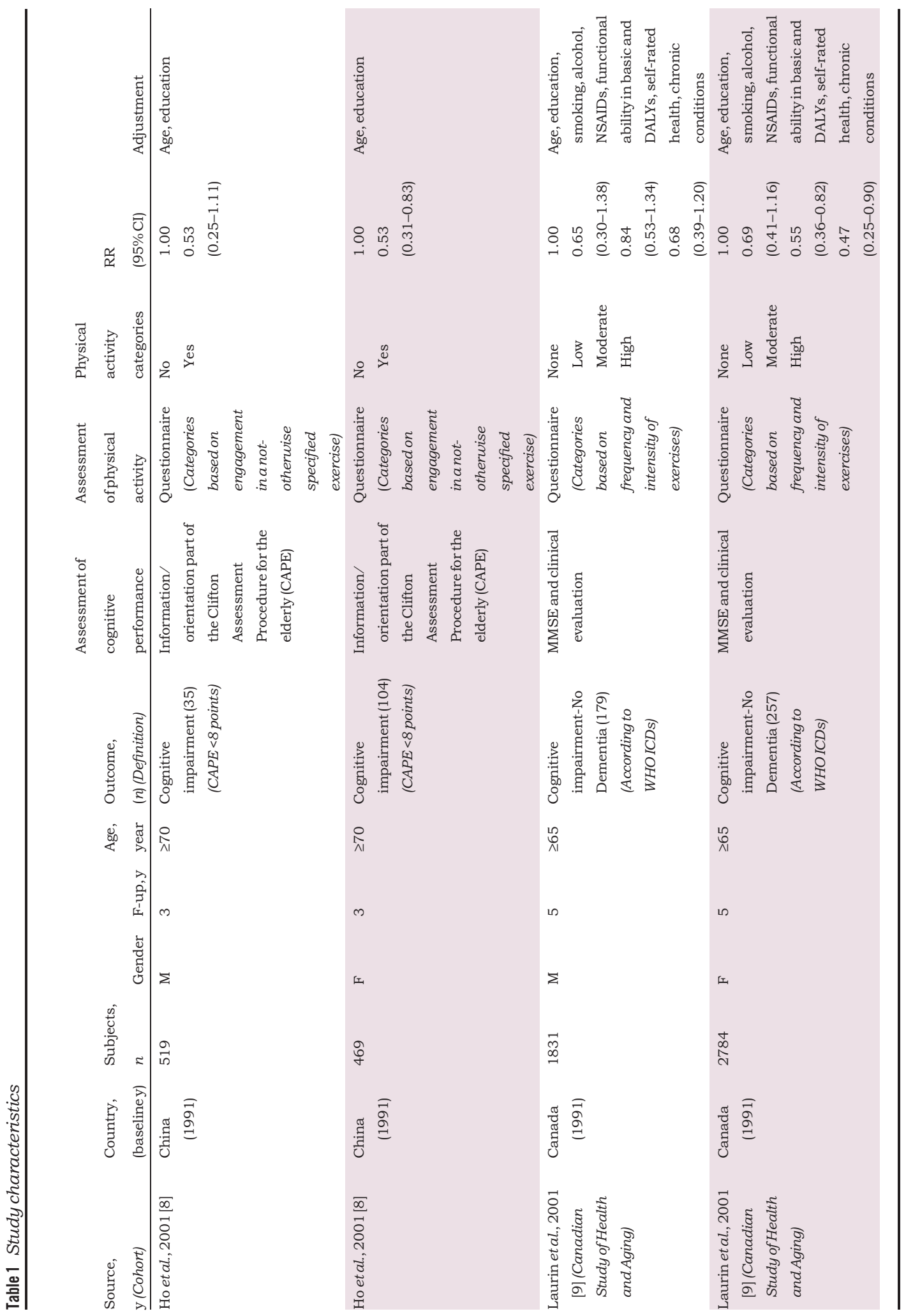




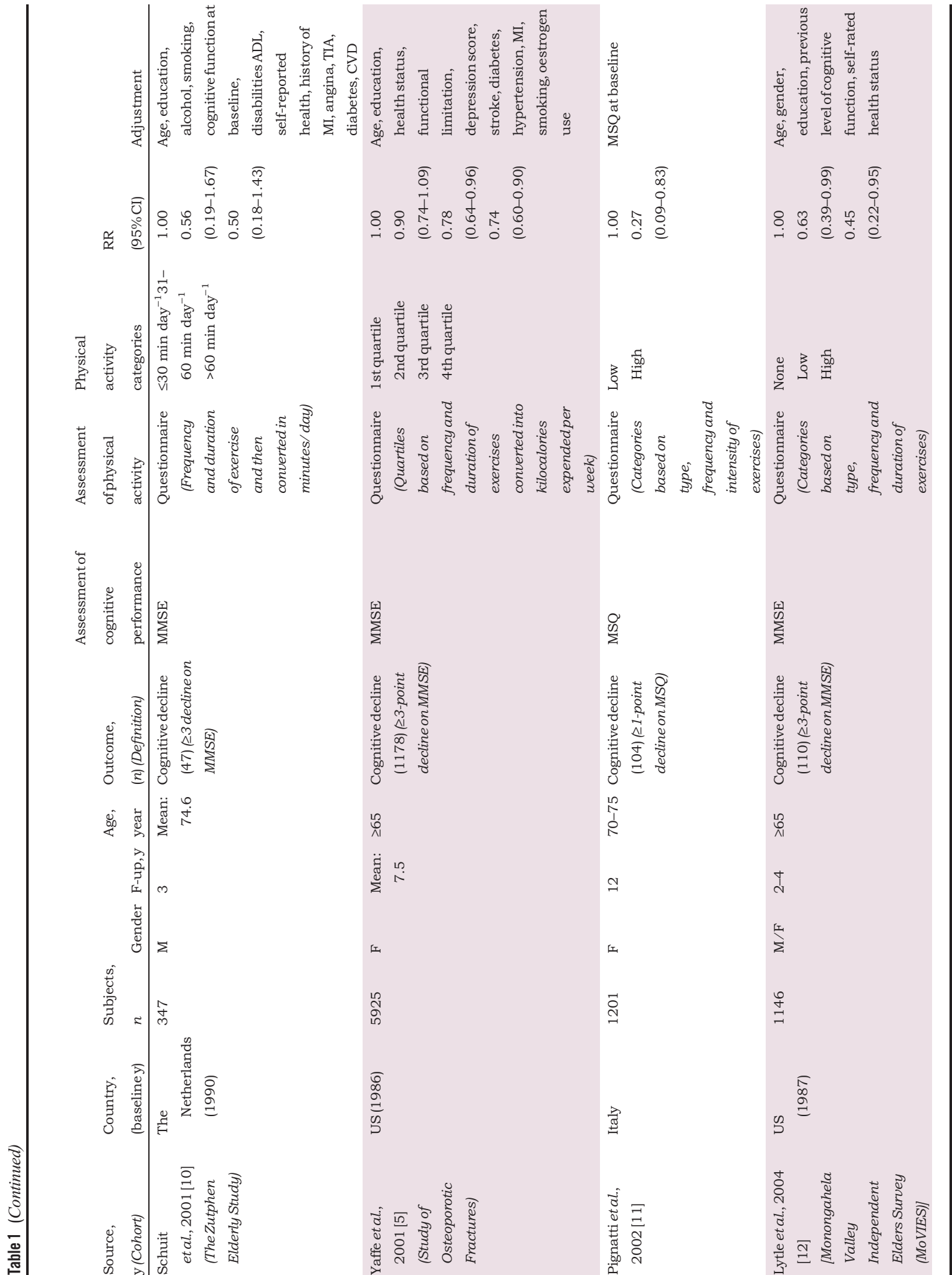




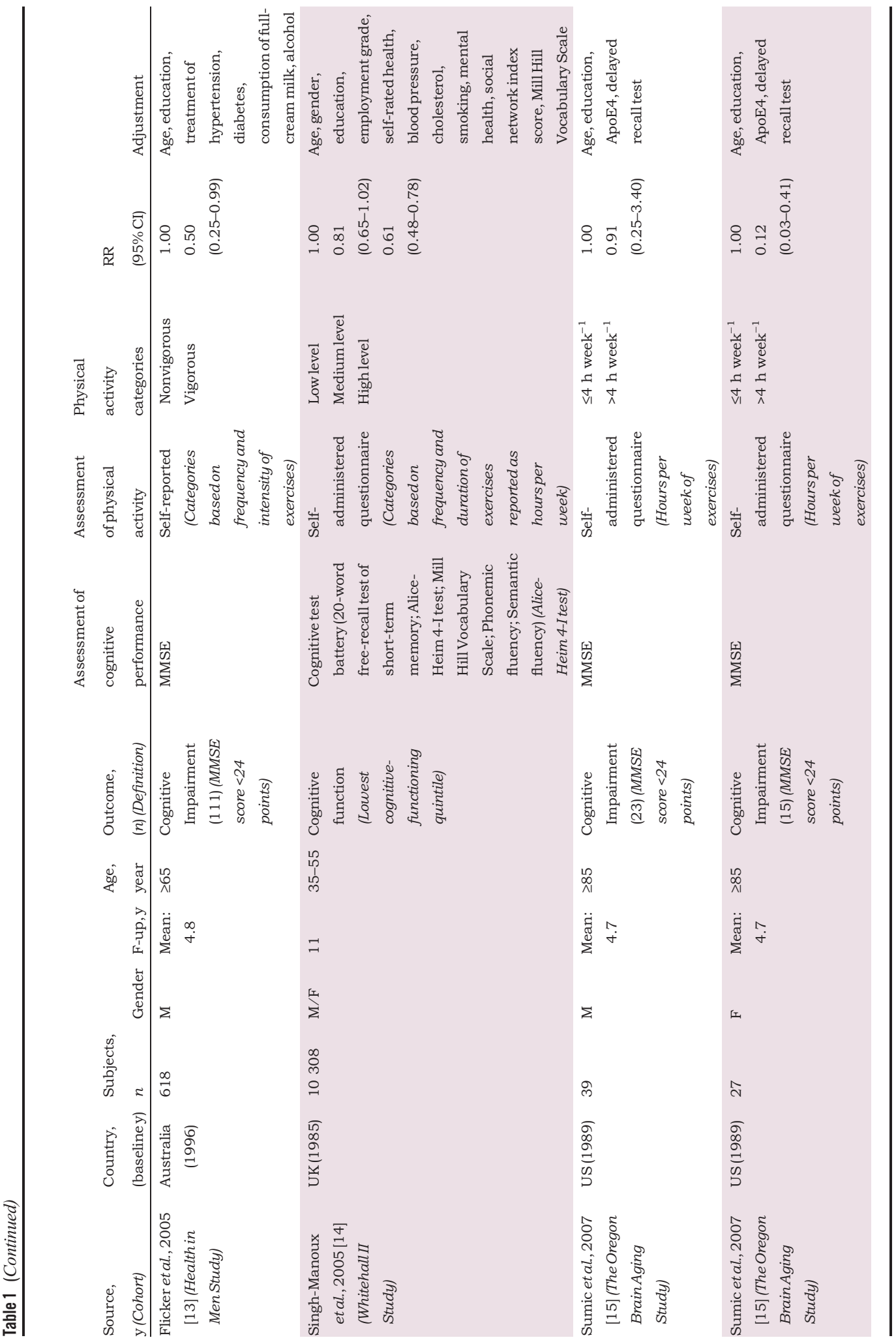




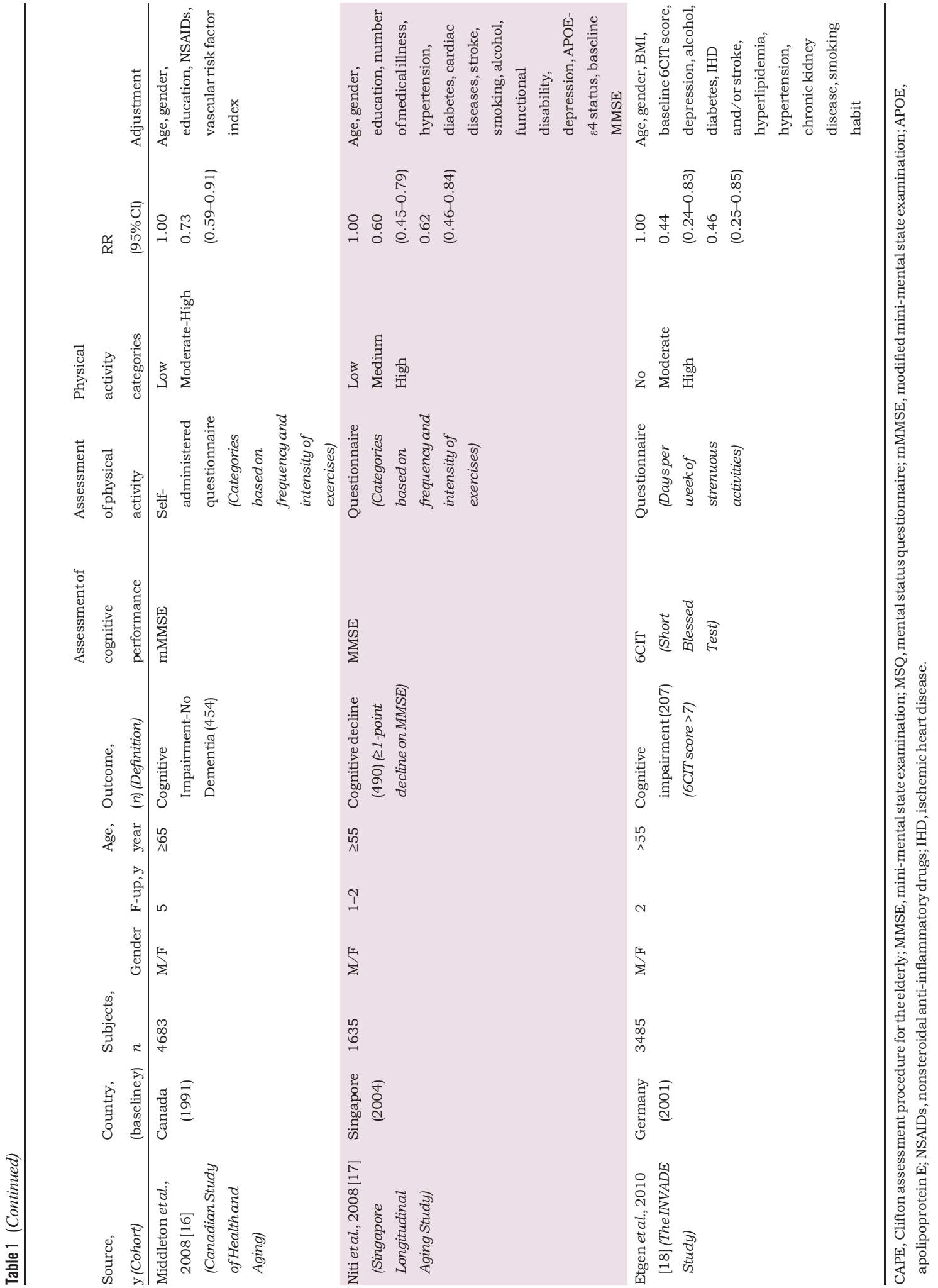


complete adjustment for potential confounders; the confounding variables included in this analysis are shown in Table 1 .

The primary aim of the present meta-analysis was to evaluate whether high levels of physical activity were associated with significant protection against cognitive decline at follow-up. Thus, for studies reporting low levels of physical activity, instead of high, in relation to cognitive decline, we recalculated the HR using conventional procedures. Statistical heterogeneity was evaluated using the $\mathrm{I}^{2}$ statistic, which assesses the appropriateness of pooling the individual study results. The $\mathrm{I}^{2}$ value provides an estimate of the amount of variance across studies because of the heterogeneity rather than chance. Where $\mathrm{I}^{2}$ was greater than $50 \%$, heterogeneity was considered to be high. Moreover, to further investigate the heterogeneity across studies, we performed sensitivity analyses by dividing studies into groups according to their main characteristics. Subgroup analyses were then performed according to gender, mean sample size of the study populations (less than/at least 3500), mean duration of follow-up (less than/at least 5 years) and method used to evaluate cognitive function (minimental state examination (MMSE)/other). Publication bias was appraised by visual inspection of the funnel plot of effect size against standard error and, analytically, by the Egger's test.

\section{Results \\ Study identification and selection}

Our search strategy yielded 58 articles (Fig. 1). Of these, we first excluded 17 articles because they had a cross-sectional, case-control or interventional design. The selected articles were then carefully reviewed, and a further 14 articles were excluded because the reported outcome was incidence of dementia or Alzheimer's disease, i.e. not the outcome of interest. Subsequently, 12 papers were excluded because they did not report estimates of the association between physical activity and decline in cognitive function. The reasons for exclusion in all cases are reported as supplementary information.

Thus, 15 prospective studies [5, 8-18] were included in the analysis. Of these, three conducted analyses separately for men and women and so were entered into the final analysis each as a single paper. The number of participants included in the studies varied from 27 to 10308 , with a follow-up time ranging from 1 to 12 years. A total of 33816 nondemented sub-
Fig. 1 Flow chart of search strategy.

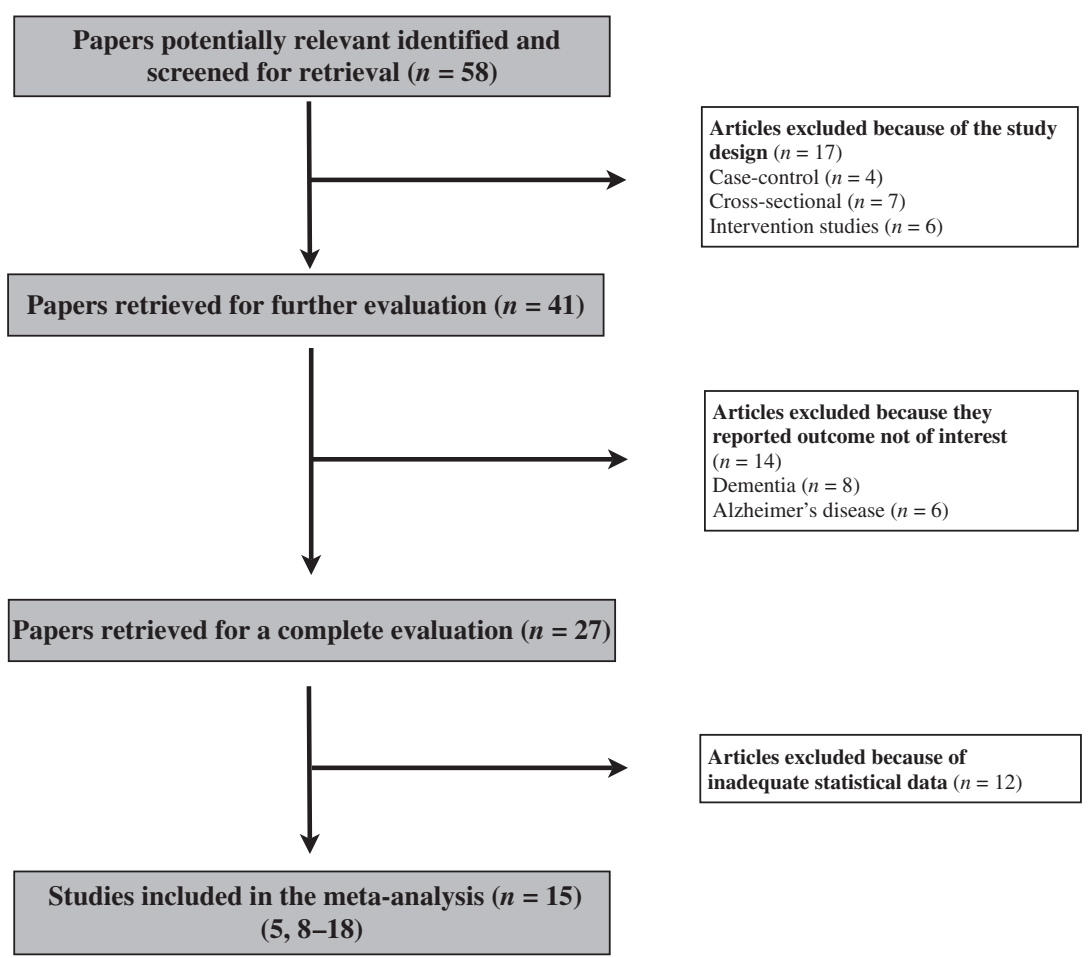


jects were included in the analysis. During the followup period, 3210 incident cases of cognitive decline were reported.

Characteristics of the studies included in the metaanalysis are summarized in Table 1 . The included studies were conducted all over the world, including China, Singapore, USA, Canada and Europe. All of the studies included only elderly subjects ( $>65$ years) with the exception of the study by Singh-Manoux et al., [14] that investigated younger subjects too. With regard to the methods used to assess cognitive functioning at baseline, most of the studies used the MMSE. In addition, the definition of cognitive decline at follow-up varied substantially in terms of points of decline for cognitive tests used to measure cognitive function.

\section{Meta-analysis}

Meta-analytic pooling under a random-effects model showed that subjects who performed physical activity at baseline had a significantly reduced risk of cognitive decline during follow-up. Indeed, by grouping studies according to the different levels of physical activity, subjects who reported performing a high level of activity had a 38\% reduced risk of cognitive decline with respect to those who reported being sedentary (HR 0.62, 95\% CI 0.54-0.70; $P<0.00001$ ) (Fig. 2). We found no significant heterogeneity amongst the studies $\left(\mathrm{I}^{2}=17 \% ; P=0.26\right)$.

Similarly, when low-to-moderate levels of physical activity were taken into consideration, the significant protection against cognitive decline during follow-up was still observed (HR 0.65, 95\% CI 0.57-0.75; $P<0.00001)$, and with no significant heterogeneity amongst the studies $\left(\mathrm{I}^{2}=33 \% ; P=0.10\right)$ (Fig. 3).

\section{Sensitivity analyses}

To investigate the possible differences across studies, we performed sensitivity analyses by grouping studies according to various characteristics such as gender of the study population, study size (mean size of the study sample was 3500), length of follow-up (mean duration was 5 years) and method used to determine cognitive function (MMSE/other). Smaller studies, including only women, and with a shorter duration of follow-up, showed a tendency towards a higher estimate of association in terms of significant reduced risk of cognitive decline, compared with larger studies, in men, and with a longer follow-up period (Table 2).

\section{Publication bias}

Funnel plots of effect size versus standard error to investigate possible publication bias were broadly symmetrical, suggesting the absence of publication bias for both high and moderate levels of physical activity $(P>0.05$ for both levels, Egger's test) (Figs 4 and 5).

\section{Discussion}

This is the first meta-analysis that aimed to investigate the association between physical activity and cognitive decline in nondemented subjects. The overall analysis of 15 cohort prospective studies investigating 30331 nondemented subjects followed for a period of 1-12 years and 3003 incident cases of cognitive decline showed that physically active individuals at baseline have a significantly reduced risk of developing cognitive decline during follow-up. Indeed, the cumulative analysis demonstrated a 38\% reduced risk of cognitive decline in subjects with high

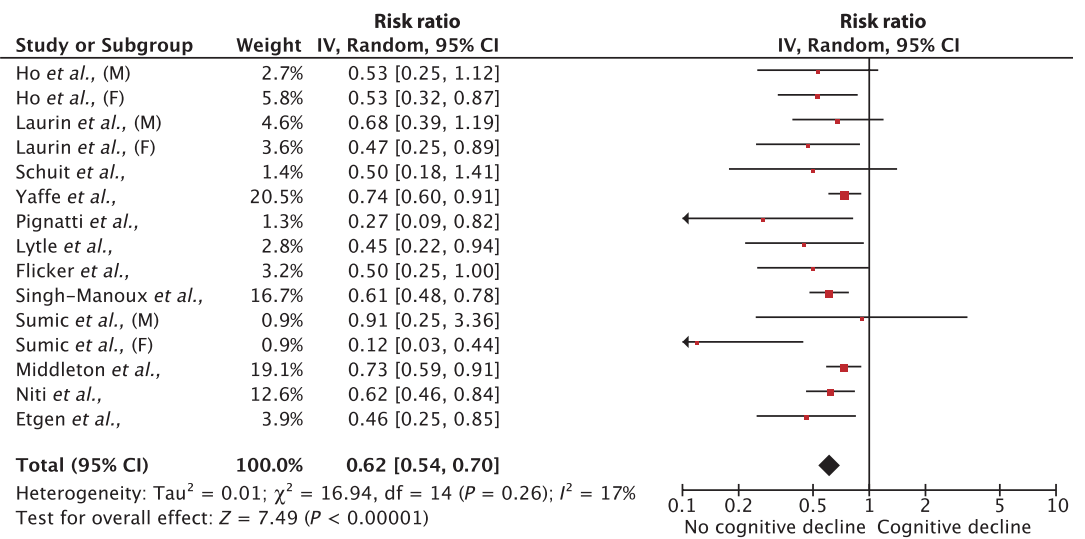

Fig. 2 Forest plot of studies investigating a high level of physicalactivity. 
Fig. 3 Forest plot of studies investigating a low-to-moderate level of physicalactivity.

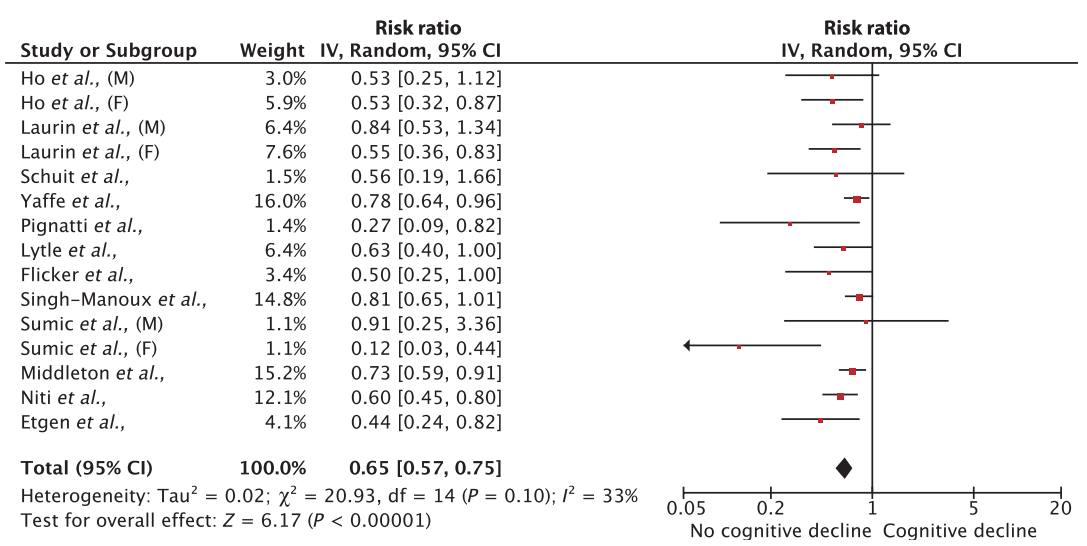

Table 2 Subgroupanalyses

\begin{tabular}{|c|c|c|c|}
\hline & Studies, $n$ & $\begin{array}{l}\text { High level of } \\
\text { physical activity }\end{array}$ & $\begin{array}{l}\text { Moderate level of } \\
\text { physical activity }\end{array}$ \\
\hline \multicolumn{4}{|l|}{ Gender } \\
\hline Males & 10 & $0.63(0.56-0.72)$ & $0.70(0.62-0.79)$ \\
\hline Females & 10 & $0.60(0.51-0.71)$ & $0.63(0.54-0.75)$ \\
\hline \multicolumn{4}{|l|}{ Sample size } \\
\hline$<3500$ subjects & 12 & $0.53(0.45-0.64)$ & $0.57(0.48-0.67)$ \\
\hline$\geq 3500$ subjects & 3 & $0.70(0.62-0.79)$ & $0.77(0.68-0.87)$ \\
\hline \multicolumn{4}{|c|}{ Duration of follow-up } \\
\hline$<5$ years & 9 & $0.54(0.44-0.65)$ & $0.55(0.46-0.66)$ \\
\hline$\geq 5$ years & 6 & $0.67(0.59-0.77)$ & $0.74(0.65-0.85)$ \\
\hline \multicolumn{4}{|c|}{ Method used to determine cognitive function } \\
\hline MMSE & 10 & $0.64(0.54-0.75)$ & $0.67(0.57-0.78)$ \\
\hline Others & 5 & $0.56(0.46-0.68)$ & $0.57(0.50-0.80)$ \\
\hline
\end{tabular}

MMSE, mini-mental state examination levels of physical exercise, compared to sedentary subjects. Moreover, low-to-moderate levels of physical activity similarly resulted in a significantly reduced risk of deterioration of cognitive performance $(-35 \%)$.

To date, few studies have investigated the relationship between an active lifestyle and cognitive performance in mentally healthy subjects, and results have been conflicting [8-18]. Recent data, including some from longitudinal studies and randomized trials, reported a significant association between physical activity during leisure time and a reduced risk of cognitive impairment at follow-up [4, 9], whereas other studies reported no significant benefit of physical activity on the decline in cognitive function [10, 19]. Recently, Hamer \& Chida [7] conducted a meta-analysis to investigate the role of physical activity on the occurrence of neurodegenerative diseases in nondemented subjects. In the overall analysis, they found that physical activity is able to decrease the risk of neurodegenerative diseases such as clinical dementia and Alzheimer's disease, but they did not take into account cognitive decline as a clinical outcome. By contrast, the present meta-analysis is the first, to the best of our knowledge, that included only cognitive decline as the clinical outcome. The choice to study healthy subjects in relation to the decline in cognitive functions was based on the hypothesis that physical activity may help cognitive performance during ageing, by preventing disability rather than a specific disease. Cognitive decline can, in fact, occur as a part of the ageing processes of the brain, without leading to dementia but resulting in a poorer quality of life. Nonetheless, the diagnosis of dementia is based on a number of parameters other than the worsening of 


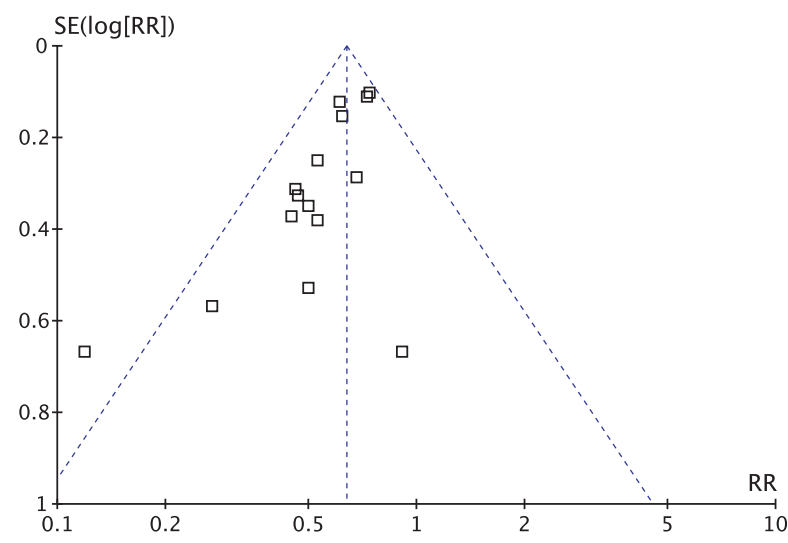

Fig. 4 Funnel plot for studies investigating a high level of physicalactivity.

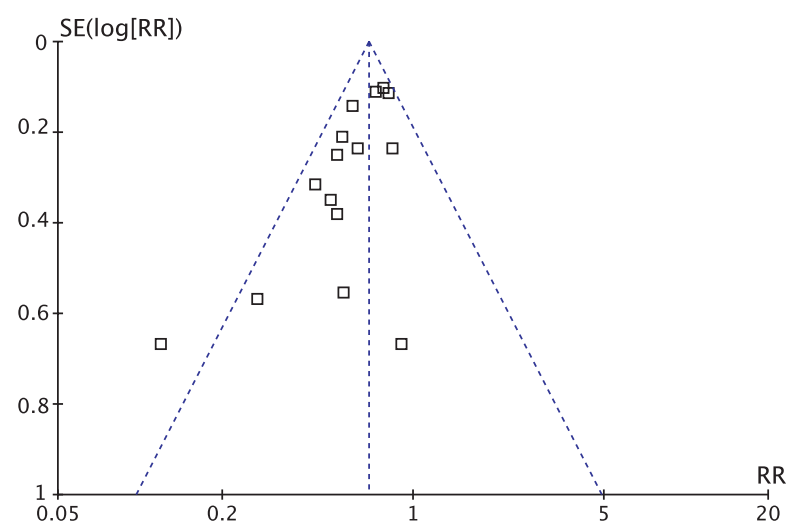

Fig. 5 Funnel plot for studies investigating a low-to-moderate level of physicalactivity.

cognitive performance, and patients referred to as 'nondemented' could show signs of slight cognitive decline as early clinical manifestations of neurodegenerative disease.

Several explanations for the protective effect of physical activity on cognitive functions have been suggested. First, physical exercise helps to maintain cerebrovascular integrity, by sustaining blood flow and the supply of oxygen and nutrients to the brain [20]. Furthermore, physical activity positively influences cardiovascular risk factors, such as diabetes, hypertension, obesity and dyslipidaemia, and reduces the incidence of cardiovascular and cerebrovascular events, with global haemodynamic benefits [21]. Secondly, another possible protective mechanism is the neurotrophic effect of physical exercise. This may stimulate the release of neurotrophins, increasing synapses and dendritic receptors, and promoting neuronal growth and survival [22]. Finally, it has been reported that an active lifestyle is able to prevent stress by reducing cortisol levels, which can positively influence cognitive function [23].

There are a few limitations in this meta-analysis. First, the methods used to investigate cognitive decline and levels of physical activity varied substantially across the included studies. The MMSE test was the most frequently used tool for the diagnosis of cognitive decline, but other tests were used in some studies. This might have resulted in a nonhomogenous definition of cognitive decline amongst the studies. Indeed, the MMSE test with the classical cut-off (>3-point decline at follow-up or a score lower than 24 points) seems to be very suitable for the diagnosis of cognitive decline but is affected by learning bias and is therefore less accurate compared to other neuropsychological tests. By contrast, however, sensitivity analysis showed no significant difference for estimates of association in relation to the different methods used to determine cognitive function. Moreover, with regard to physical activity, data were obtained from questionnaires; thus, bias could be introduced by misinterpretation of the questions and the personal perception of fatigue. In addition, studies differ in the methods used to classify the level of activity, ranging from studies with a simple differentiation of active/not active to others with three or four levels of intensity. Nevertheless, heterogeneity results as well as subgroup analyses did not show any significant differences in risk reduction amongst physically active subjects in terms of the intensity of activity. Indeed, in the overall results, we did not observe a doseresponse effect; instead, we found similar estimates of association for both high and low-to-moderate intensity of exercise.

In conclusion, these results highlight the important role of physical activity in the protection of mental functions even in subjects without neurodegenerative disease. These considerations could be important especially because the population is ageing and a good cognitive function is fundamental for individual autonomy and quality of life, even in nondemented subjects. The effect of physical activity does not appear to be dose dependent, but may be stronger in women than in men. However, further studies are needed to determine the optimal type, frequency and intensity of exercise to preserve the integrity of cognitive function. 


\section{Conflict of interest statement}

No conflict of interest was declared.

\section{References}

1 A guide for population-based approaches to increasing levels of physical activity. Implementation of the WHO global strategy on diet, physical activity, and health. Geneva, Switzerland: World Health Organization, 2007 (Available at http://www.who.int/dietphysicalactivity/pa/en/index.html).

2 Jernigan TL, Archibald SL, Fennema-Notestine C et al. Effects of age on tissues and regions of the cerebrum and cerebellum. NeurobiolAging 2001; 22: 581-94.

3 Wise RA. Dopamine, learning and motivation. Nat Rev Neurosci 2004; 5: 483-94.

4 Weuve J, Kang JH, Manson JE, Breteler MMB, Ware JH, Grodstein F. Physical activity, including walking, and cognitive function in older women. JAMA 2004; 292: 1454-561.

5 Yaffe K, Barnes D, Nevitt M, Lui LY, Covinsky K. A prospective study of physical activity and cognitive decline in elderly women: women who walk. ArchIntern Med 2001; 161: 1703-8.

6 Dishman RK, Berthoud HR, Booth FW et al. Neurobiology of exercise. Obesity 2006; 14: 345-56.

7 Hamer M, Chida Y. Physical activity and risk of neurodegenerative disease: a systematic review of prospective studies. Psychol Med 2009; 39: 3-11.

8 Ho SC, Woo J, Sham A, Chan SG, Yu AL. A 3-year follow-up study of social, lifestyle and health predictors of cognitive impairment in a Chinese older cohort. Int J Epidemiol 2001; 30: 138996.

9 Laurin D, Verreault R, Lindsay J, MacPherson K, Rockwood K. Physical activity and risk of cognitive impairment and dementia in elderly persons. Arch Neurol2001; 58: 498-504.

10 Schuit AJ, Feskens EJ, Launer LJ, Kromhout D. Physical activity and cognitive decline, the role of the apolipoprotein e 4 allele. Med SciSports Exerc2001; 33: 772-7.

11 Pignatti F, Rozzini R, Trabucchi M. Physical activity and cognitive decline in elderly persons. Arch Intern Med 2002; 162: 361-2.

12 Lytle ME, Vander Bilt J, Pandav RS, Dodge HH, Ganguli M. Exercise level and cognitive decline: the MoVIES project. Alzheimer Dis Assoc Disord 2004; 18: 57-64.

13 Flicker L, Almeida OP, Acres J et al. Predictors of impaired cognitive function in men over the age of 80 years: results from the Health in Men Study. Age Ageing 2005; 34: 77-80.

14 Singh-Manoux A, Hillsdon M, Brunner E, Marmot M. Effects of physical activity on cognitive functioning in middle age: evidence from the Whitehall II prospective cohort study. AmJ Public Health 2005; 95: 2252-8.
15 Sumic A, Michael YL, Carlson NE, Howieson DB, Kaye JA. Physical activity and the risk of dementia in oldest old. J Aging Health 2007; 19: 242-59.

16 Middleton L, Kirkland S, Rockwood K. Prevention of CIND by physical activity: different impact on VCI-ND compared with MCI. JNeurol Sci2008; 269: 80-4.

17 Niti M, Yap KB, Kua EH, Tan CH, Ng TP. Physical, social and productive leisure activities, cognitive decline and interaction with APOE-epsilon 4 genotype in Chinese older adults. Int Psychogeriatr2008; 20: 237-51.

18 Etgen T, Sander D, Huntgeburth U, Poppert H, Forstl H, Bickel H. Physical activity and incident cognitive impairment in elderly persons. The INVADE study. Arch Intern Med 2010; 170: 18693.

19 Madden DJ, Blumenthal JA, Allen PA, Emery CF. Improving aerobic capacity in healthy older adults does not necessarily lead to improved cognitive performance. PsycholAging 1989; 4: 307-20.

20 Chodzko-Zajko WJ, Moore KA. Physical fitness and cognitive functioning in aging. Exerc Sport SciRev 1994; 22: 195-220.

21 Sofi F, Capalbo A, Marcucci R et al. Leisure time but not occupational physical activity significantly affects cardiovascular risk factors in an adult population. Eur J Clin Invest 2007; 37: 94753.

22 Gomez-Pinilla F, So V, Kesslak JP. Spatial learning and physical activity contribute to the induction of fibroblast growth factor: neural substrates for increased cognition associated with exercise. Neuroscience 1998; 85: 53-61.

23 Kalmijn S, Launer LJ, Stolk RP et al. A prospective study on cortisol, dehydroepiandrosterone sulfate, and cognitive function in the elderly. JClin Endocrinol Metab 1998; 83: 3487-92.

Correspondence: Francesco Sofi, MD, PhD, Department of Medical and Surgical Critical Care, Thrombosis Centre, University of Florence, Viale Morgagni 85, 50134, Florence, Italy.

(fax: +39-055-7949418; e-mail: francescosofi@gmail.com).

\section{Supporting Information}

Additional Supporting Information may be found in the online version of this article:

\section{Data S1. Reasons of exclusion.}

Please note: Wiley-Blackwell are not responsible for the content or functionality of any supporting materials supplied by the authors. Any queries (other than missing material) should be directed to the corresponding author for the article. 